\title{
A Novel Biomarker TLCD1 Correlates with Prognosis and Immune Infiltrates in Hepatocellular Carcinoma
}

\section{Hanyu Shen}

Nantong University

Ailong Huang

Nantong University

Dandan Zhu

Nantong University

Jiali Zhang

Nantong University

\section{Shiqi Ren}

Department of Clinical Biobank, Affiliated Hospital of Nantong University, Nantong, Jiangsu 226000, People's Republic of China

Lian Duan

Nantong University

\section{Pingping Sun}

Department of Clinical Biobank, Affiliated Hospital of Nantong University, Nantong, Jiangsu 226000, People's Republic of China

Ziheng Wang ( 1517073031@xlxy.ntu.edu.cn )

Affiliated Hospital of Nantong University https://orcid.org/0000-0001-8873-732X

\section{Yinong Duan}

Nantong University

\section{Research}

Keywords: TLDC1, HCC, TCGA, fatty acid metabolism, TME, Tregs

Posted Date: July 16th, 2020

DOI: https://doi.org/10.21203/rs.3.rs-42288/v1

License: (c) (1) This work is licensed under a Creative Commons Attribution 4.0 International License.

Read Full License 


\section{Abstract}

Background: The HCC has seen a spike in the morbidity and the mortality rate in recent times. This calls for an urgent understanding of the underlying molecular mechanisms of the HCC and to speed up the quest for the search of the target molecules to ensure quick diagnosis and prognosis subsequently. TLCD1 is a gene only reported in membrane fluidity. The variations in the TLCD1 expression levels related to the infiltration of the immune cells in HCC and the prognosis shall be examined initially.

Methods: The data received from TCGA shall provide the details of the gene expression, clinicopathology analysis and TME estimate, along with the enrichment analysis. Moreover, we performed additional analysis of the bioinformatics available. The immune responses of TLCD1 expression in HCC were analyzed using CIBERSORT and TIMER, while the statistical analysis was handled through R. HPA was used to validate the outcomes.

Results: Higher TLCD1 expression is strongly correlated with a poor prognostic and worse overall survival. Specifically, the increase in TLCD1 expression positively correlated with Tregs cells and T cells CD4 memory resting. The pathways strongly associated with TLCD1 was fatty acid metabolism and PPAR signaling pathway.

Conclusions: From the outcome of the study, it could be surmised that TLCD1 could be considered as a potential target for future treatment of HCC, as it was observed to be associated with the tumorinfiltrating immune cells in tumor microenvironment, establishing itself as a novel potential prognostic biomarker in HCC.

\section{Background}

Liver cancer is one of the most common cancer with a rate of high mortality around the world. In the cancer of the liver, the HCC is the most common type, with an increasing trend of incidence presently ${ }^{1,2}$. China accounts for about $50 \%$ of the morbidity number of liver cancer in the world ${ }^{2}$. For the HCC patients till now, early diagnosis such as the B-mode, CT scan and the serum AFP have been the common tools ${ }^{3,4}$. Despite the developments in the detection and management of $\mathrm{HCC}$, patients with $\mathrm{HCC}$ remain suffered a bad life for current therapies ${ }^{5}$. Hence, it becomes necessary to understand the underlying molecular mechanisms of HCC and identify the target molecules to assist quick diagnosis and prognosis.

TLCD1 is a protein coding gene. The human and mouse TLCD1 gene expression is evident in a variety of tissues, with muscle, heart, fat and liver being the strongest for TLCD1 presence. Using a series of careful immune-detection experiments and the Myc-epitope tagging mechanisms, the TLCD1 was initially found in the plasma membrane of the mammalian cells ${ }^{6}$. Its action at the level of the plasma membrane in limiting the amounts of LCPUFA-containing phospholipids was proved in the previous study by its localization and the effect on PUFA content in membrane phospholipids ${ }^{7}$. In human physiology the LCPUFA are substances with a range of important structural and regulatory functions ${ }^{8}$. LCPUFA are 
involved in numerous biological processes and are major components of complex lipid molecules 9,10 . Liver is an important place of lipid metabolism and lipid is a crucial component of membrane. lipid destruction can reflect their important roles during tumor initiation and disease progression at different points such as disruption of normal tissue architecture, cancer cell migration, interaction of cancer cells with components of the tumor stroma and lipid metabolic reprogramming in cancer cells ${ }^{11-14}$. Lipid alterations which might be involved in the onset of cancer and its development, such as lung cancer ${ }^{15}$, breast cancer ${ }^{16,17}$, lung cancer ${ }^{15}$ and colon cancer ${ }^{18}$. When TLCD1 was removed more unsaturated fats were incorporated, thereby leaving the membranes in a healthy restored state, albeit the excess saturated fat being the medium in which the cells were being grown? ${ }^{7}$.

HCC is a common type of cancer. However, the role of TLCD1 in HCC remains unknown. In the research, we explored the expression of TLCD1 in human HCC samples founded on microarray data that we downloaded from the TCGA database. Increasing evidence indicates that tumorigenesis is often triggered in a tumor microenvironment (TME), which is significantly influence the gene expression of tumor cells,TME composed extracellular matrix, blood or lymphatic vessels, fibroblasts, immune cells and inflammatory cells ${ }^{19}$. Stromal and immune cells are two main types of nontumor components in the $\mathrm{TME}$, and the investigation of their interaction has been valuable for developing innovative HCC-directed immunotherapies. In this study, we calculated the stromal and immune scores of HCC cohorts from the TCGA database by applying the ESTIMATE algorithm and investigate the relationship between TME cells and TLCD1 expression in patients with $\mathrm{HCC}^{20}$. Meanwhile, we used R language (Version 3.5.3) and statistical analysis to examine the correlation of TLCD1 expression with clinical parameters as well as the prognosis in patients with HCC. GEPIA and ICGA data were used to confirm the relationship between TLCD1 and overall survival. Moreover, we performed an evaluation of the landscape of TME and TLCD1 in HCC. To delve deeper into the biological processes involved in the pathogenesis of HCC associated with the TLCD1 regulatory network, we performed the GSEA along with the enhanced GO and KEGG analyses.

\section{Materials And Methods}

\section{Gene Expression Analysis}

The TCGA official website for the liver provided the pertinent gene expression data (424 files, Workflow Type: HTSeq-FPKM ${ }^{21}$. Certain customizable functions were made available from the GEPIA online database (http://gepia.cancer-pku.cn/). Adopting a standard processing pipeline the RNA sequencing expression data of 8,587 normal samples and 9,736 tumors from the GTEx and the TCGA projects were analyzed through GEPIA ${ }^{22}$. The TCGA database provided the normal and tumor samples in the GEPIA database. To determine the differential expression of TLCD1, Boxplot, using the disease state as a variable, was graphed.

\section{Survival analysis and prognosis analysis}


The TCGA official website for the liver provided the data of systematic analysis of immune infiltrates and the clinical information (377cases, Data Type: Clinical Supplement). The cases in the TNM stage, distant metastasis, lymph node metastasis, local invasion, overall survival time, and insufficient or missing data on age, were excluded. ICGC The clinical data was further analyzed and retained. The TCGA provided the guidelines for the publication of this study. The GEPIA database computed the correlations of the disease-free survival rate with the TLCD1 expression in HCC. To further analysis the relationship between the survival days of HCC patients and the expression of high degree TLCD1, a total of 203 patients identified and acquired from ICGC dataset(https://icgc.org/) were chosen for validation.

\section{TME estimate}

We used the single-sample gene-set enrichment analysis algorithm to quantify the relative abundance of each cell infiltration in the HCC TME and the stromal and immune scores were calculated by applying the ESTIMATE package to the downloaded RNA expression data. We combine the TLCD1 with the TME cells for further analysis ${ }^{23}$.

\section{Immune Infiltrates Analysis}

The relationships between the possible tumor-infiltrating immune cells and the expression of TLCD1 was evaluated with the correlation module of TIMER, an efficient resource helping in the systematic analysis of the immune infiltrates across several types of cancer (https://cistrome.shinyapps.io/timer/) ${ }^{22}$. To determine the abundance of tumor-infiltrating immune cells from gene expression profiles, a previously published statistical deconvolution method was applied by TIMER ${ }^{24}$. The abundance of immune infiltrates could be estimated from the TIMER database that included 10,897 samples across 32 cancer types from TCGA. The correlation of TLCD1 expression with the abundance of immune infiltrates, including the dendritic cells, neutrophils, macrophages, CD8 + T cells, CD $4+T$ cells, through the gene modules, and the TLCD1 expression in liver cancer was duly analyzed. The left-most pane displays the gene expression levels against the tumor purity ${ }^{25}$. Moreover, a deconvolution algorithm based on gene expression, CIBERSORT (http://cibersort.stanford.edu/), can evaluate the changes in the expression of all the sets of the other genes in the sample against one specific set of genes. In the current analysis, via CIBERSORT, the immune response of 22 immune infiltrates cells in HCC, for determining its correlation with the molecular subpopulation and survival, was gauged. The gene expression datasets were uploaded to CIBERSORT web portal using the standard annotation files with the algorithm running at 1,000 permutations, its default signature matrix. Establishing a measure of confidence in the results a pvalue for deconvolution through the Monte Carlo sampling was estimated by CIBERSORT. We used 374 tumor samples from the TCGA divided into 2 groups in order to assess the influence of TLCD1 expression in the immune microenvironment. To select the lymphocyte possibly affected by the expression of TLCD1 the $p$-value $<0.05$ was set as the criterion.

\section{Gene Set Enrichment Analysis}

GSEA was performed using normalized RNA-Seq data by TCGA ${ }^{26}$. The annotated gene sets of c5.all.v7.0.symbols.gmt and c2.cp.kegg.v7.0.symbols.gmt in the Molecular Signatures Database 
(MSigDB) were selected in GSEA version 3.0. The number of permutations was set at 1,000 to determine the normalized enrichment score. GO terms, KEGG pathways were performed to explore the potential biological functions of TLCD1 by using GSEA. Enrichment results satisfying a nominal P-value $<0.05$ and a false discovery rate FDR q-value $<0.25$ were considered statistically significant.

\section{Human Protein Atlas}

The human protein atlas database (HPA) (www.proteinatlas.org) provides access to 32 human tissues and their protein expressions by using antibody profiling to accurately assess protein localization. ${ }^{27}$ Additionally, the HPA provides measurements of RNA levels. HPA database was used to validate protein expression of TLCD1 between normal and liver cancer tissues.

\section{Statistical Analysis}

The R language (Version 3.5.3) conducted the download of the statistical analyses from TCGA. To calculate the $95 \% \mathrm{Cl}$ and the HR the multivariate Cox and the Univariate proportional hazards models were utilized. The comparison of several clinical characteristics with survival was done using the Univariate survival analysis. To evaluate the influence of TLCD1 expression and other clinical pathological factors (lymph node, distant metastasis, tumor status, grade, gender, and age) on survival, the Multivariate Cox analysis was conducted. The cut-off criterion was set with the $P$-value of TLCD1 expression $<0.05$. Using the logistic regression, the correlations between the TCLD1 expression and the clinical characteristics were analyzed.

\section{Results}

\section{TLCD1 expression is significantly upregulated in HCC}

The TLCD1 mRNA levels in the normal and the tumor tissues of liver cancer type were analyzed by using TCGA database, in order to determine the differences between the TLCD1 expression in the normal and tumor tissues. 50 normal files along with 374 tumor files were transformed to convert count data to values more consistent with the microarray results. The boxplot displayed the expression of TLCD1 between the HCC and the normal data (Fig. 1A). A significantly higher TLCD1 expression was revealed in the tumor tissues ( $p$-value $=1.017 \mathrm{e}-24)$ by this analysis. Whereas, a significantly increased TLCD1 mRNA expression in HCC compared normal group with liver cancer group ( $p$-value $<0.010$, |Log2FC| $>1$ ) (Fig. 1B) was found using the GEPIA database.

\section{Relationship between TLCD1 expression and clinical characteristics}

$\chi 2$ tests revealed the relationship between the TLCD1 expression and the clinical characteristics (Table.1). To investigate the association with multivariable characteristics and tumor progression in TCGA patients, we using cox regression(Table.2). Univariate analysis of correlation revealed that some factors, including 
pathological stage $(H R=1.865, p$-value $<0.001)$, tumor $(H R=1.804, p$-value $<0.001)$ along with the expression of TLCD1 $(H R=1.036, p$-value $=0.003)$ are significantly associated with tumor development. In multivariate analysis as a forest boxplot was observed in Fig. $1 \mathrm{C}$, the TLCD1 ( $p$-value $=0.041$ ) expression is an independent prognostic factor for tumor progression.

Table 1. Clinical Characteristics of the Patients at Baseline.

\begin{tabular}{|c|c|c|c|c|c|}
\hline characteristic & $\mathrm{n}$ & low & high & Pearson $x^{2}$ & $\mathrm{p}$ \\
\hline total & 234 & 117 & 117 & & \\
\hline \multicolumn{6}{|l|}{ age } \\
\hline$\leq 60$ & 130 & 58 & 72 & 3.3923 & 0.0655 \\
\hline$>60$ & 104 & 59 & 45 & & \\
\hline \multicolumn{6}{|l|}{ gender } \\
\hline male & 160 & 82 & 78 & 0.3162 & 0.57389 \\
\hline female & 74 & 35 & 39 & & \\
\hline \multicolumn{6}{|l|}{ grade } \\
\hline I & 29 & 18 & 11 & 10.2567 & 0.01651 \\
\hline II & 103 & 59 & 44 & & \\
\hline III & 92 & 38 & 54 & & \\
\hline IV & 10 & 2 & 8 & & \\
\hline \multicolumn{6}{|l|}{ stage } \\
\hline I & 113 & 66 & 47 & 7.8372 & 0.0494 \\
\hline II & 49 & 19 & 30 & & \\
\hline III & 67 & 31 & 36 & & \\
\hline IV & 5 & 1 & 4 & & \\
\hline \multicolumn{6}{|l|}{ tumor } \\
\hline I & 115 & 67 & 48 & 14.246 & 0.002588 \\
\hline II & 51 & 20 & 31 & & \\
\hline III & 58 & 27 & 31 & & \\
\hline IV & 10 & 3 & 7 & & \\
\hline
\end{tabular}


Table 2. Association between TLCD1 expression and clinicopathologic characteristics using logistic regression.

\begin{tabular}{|lll|}
\hline Clinical characteristic & Odds ratio (OR.95L-OR.95H) & P-Value \\
\hline Age & $0.69(0.45-1.04)$ & 0.08 \\
\hline Grade (II vs I) & $1.60(0.86-3.05)$ & 0.14 \\
\hline Grade (III vs I) & $2.78(1.45-5.49)$ & 0.00 \\
\hline Grade (IV vs I) & $9.47(2.22-65.89)$ & 0.01 \\
\hline Grade (I,II vs III,IV) & $2.12(1.38-3.29)$ & 0.00 \\
\hline Stage (II vs I) & $2.00(1.18-3.43)$ & 0.01 \\
\hline Stage (III vs I) & $1.60(0.95-2.72)$ & 0.08 \\
\hline Stage (IV vs I) & $5.44(0.79-107.72)$ & 0.13 \\
\hline Tumor (III vs I) & $2.09(1.26-3.52)$ & 0.00 \\
\hline
\end{tabular}

\section{Relationship between TLCD1 expression and poor overall survival}

To discover the associations with TLCD1 expression and overall survival in HCC patients, we firstly validated by TCGA datasets, as shown in Fig. 2A. Patients with higher TLCD1 expression had particularly shorter OS ( $P=0.019)$, Furthermore, we used GEPIA database find high levels of TLCD1 mRNA also strongly correlated with a worse survival ( $p$-value $=0.0035)(F i g .2 B)$. Patients obtained from ICGC database was also used to validate the group of high TLCD1 expression will lead to poor overall survival. The result was considered statistically significant ( $p$-value < 0.001) (Fig. 2C). Univariate analysis using logistic regression revealed that TLCD1 expression was associated with poor prognostic clinicopathologic characteristics. Increased TLCD1 expression in HCC as significantly associated with grade (III vs I, $p$-value $=0.00$; IV vs I, $p$-value $=0.01$ ), stage (II vs I, $p$-value $=0.01)$, tumor status (III vs I, $p$ value $=0.00$ ). These results suggested that liver cancer patients with high TLCD1 expression are more susceptible to a more advanced grade, stage and tumor status than those with low TLCD1 expression (Table.3). 
Table 3. Correlation between overall survival and multivariable characteristics in TCGA patients via (a) Cox regression (b) Multivariate survival model.

\begin{tabular}{|lllll|}
\hline characteristic & HR & HR.95L & HR.95H & pvalue \\
age & 1.005 & 0.987 & 1.023 & 0.591 \\
\hline gender & 1.282 & 0.801 & 2.053 & 0.301 \\
\hline grade & 1.017 & 0.746 & 1.387 & 0.914 \\
\hline stage & 1.865 & 1.456 & 2.388 & 0.000 \\
\hline T & 1.804 & 1.434 & 2.270 & 0.000 \\
M & 3.850 & 1.207 & 12.281 & 0.023 \\
\hline N & 2.022 & 0.494 & 8.276 & 0.328 \\
\hline TLCD1 & 1.036 & 1.012 & 1.060 & 0.003 \\
\hline
\end{tabular}

\begin{tabular}{|lllll|}
\hline characteristic & HR & HR.95L & HR.95H & pvalue \\
age & 1.008 & 0.989 & 1.028 & 0.392 \\
\hline gender & 1.003 & 0.601 & 1.674 & 0.992 \\
\hline grade & 1.063 & 0.766 & 1.475 & 0.713 \\
\hline stage & 0.895 & 0.333 & 2.407 & 0.826 \\
\hline T & 1.961 & 0.808 & 4.760 & 0.137 \\
M & 0.975 & 0.256 & 3.710 & 0.971 \\
\hline N & 2.519 & 0.399 & 15.904 & 0.326 \\
\hline TLCD1 & 1.026 & 1.001 & 1.051 & 0.041 \\
\hline
\end{tabular}

\section{Correlation between TLCD1 and the landscape of TME in HCC}

We first used the single sample GSEA algorithm to depict the enrichment landscape of the abundance of each cell infiltration in the HCC TME, as well as enrichment scores generated from each sample were fully clustered by hierarchical clustering method (Fig. 3A). Moreover, we proceed to excavate the relationships between TLCD1, stromal cells and immune cells, as shown in Fig. 3B\&3C. These results indicated that the expression of TLCD1 was associated with tumor microenvironment components (stromal and immune cells). 


\section{Correlation between TLCD1 expression and tumor- infiltrating immune cells}

It was aptly established that tumor-infiltrating lymphocytes were an independent predictor of survival and the sentinel lymph node status in cancers ${ }^{28}$. Hence, whether TLCD1 expression was correlated with the immune infiltration levels in liver cancer was investigated. The correlations of TLCD1 expression with the immune infiltration levels in liver cancer was assessed from TIMER. It was observed that TLCD1 expression had positive correlations with dendritic cells ( $p$-value $=3.67 \mathrm{e}-2)$, macrophages $(p$-value $=$ $2.09 \mathrm{e}-4)$ and $B$ cell $(p$-value $=4.04 \mathrm{e}-5)$ as indicated in Fig. 4A. A specific role in the immune infiltration in liver cancer was played by the TLCD1 as evidenced from the findings. Besides, we examined if the TLCD1 expression was associated with immune infiltration in the liver cancer cases. According to TLCD1 expression, the 374 tumor samples were divided into 2 parts. Overall, the screening criteria was met by the 187 samples of low and high expression groups. To infer the density of 22 types of immune cells and to explore the gene expression profiles of the downloaded samples, the established computational resource CIBERSORT was used. The assessment of the differing concentrations in the low and high TLCD1 expression groups of the 22 immune cell subtypes was done by applying the CIBERSORT algorithm. The results were exhibited in Fig. 4B. T cells CD4 memory resting, T cells follicular helper, $T$ cells regulatory (Tregs), Monocytes, Macrophages M0, Macrophages M2, and Mast cells resting were affected by TLCD1 expression.

We observed considerable differences in T cells CD4 memory resting and Tregs, macrophages and mast cells between high group and low group. Afterwards, compared with low expression group, Tregs apparently increased ( $p$-value $<0.001)$ in high expression group. Moreover, as shown in Fig. $4 \mathrm{C}$ the correlations between the 22 types of immune cells were compared as a correlation heat map. The outcome revealed that the different tumor-infiltrating immune cells subpopulations ratios were moderate to weakly correlated.

\section{Excavate correlating Tregs markers}

The relation between TLCD1 and Tregs gene markers in the liver tissue was determined using the correlation module of GEPIA Pearson correlation analysis. TNFRSF18, IL1R2, JAK1, CTLA4, IL1R1, TNFRSF 4 and CD274 are serve as potential biomarkers of Tregs. We analyzed the relationship between these metabolic genes and TLCD1 expression (high VS low), as shown in Fig. 5A, The correlation between TLCD1 expression and biomarkers expression in the TCGA database in Fig. 5B.

\section{GO and KEGG pathway analysis}

To explore the potential biological functions and to study the regulatory mechanism of the TLCD1, the KEGG pathways and GO terms were performed using GSEA. In the enrichment of the KEGG pathways and the $\mathrm{GO}$ terms the GSEA revealed significant differences (FDR $<0.25, p$-value $<0.050$ ). We selected the most significantly enriched signaling pathways based on their normalized enrichment score (NES). As shown in Table.4, the GO annotation in high TLCD1 expression resulted five negative correlated parts: 
protein activation cascade, vitamin B6 binding, retinoic acid metabolic process, cellular amino acid catabolic process and fatty acid catabolic process. The results revealed that the biological processes and molecular functions strongly associated with TLCD1 was fatty acid catabolic process, as shown in Fig. 6A. The KEGG pathway analysis showed the TLCD1 was significantly enriched in five negative pathways: tryptophan metabolism, fatty acid metabolism, drug metabolism cytochrome p450, retinol metabolism and PPAR signaling pathway, as shown in Fig. 6B. It was indicated that the metabolism pathways were strongly associated with TLCD1. In HCC patients all these functions and mechanisms are critically important.

Table 4. Signaling pathways most significantly correlated with TLCD1 expression based on their normalized enrichment score (NES) and p-value.

\begin{tabular}{|lllll|}
\hline \multirow{2}{*}{ GO } & NAME & NES & NOM p-val & FDR q-val \\
\cline { 2 - 5 } & tryptophan metabolism & -2.20 & 0.00 & 0.00 \\
\cline { 2 - 5 } & fatty acid metabolism & -2.06 & 0.00 & 0.00 \\
\hline drug metabolism cytochrome p450 & -2.03 & 0.00 & 0.00 \\
\hline KEGG & -2.03 & 0.00 & 0.00 \\
\cline { 2 - 5 } & retinol metabolism & -1.87 & 0.00 & 0.01 \\
\hline PPAR signaling pathway & -2.19 & 0.00 & 0.00 \\
\cline { 2 - 4 } & vitamin B6 binding & -2.16 & 0.00 & 0.00 \\
\hline retinoic acid metabolic process & -2.11 & 0.00 & 0.01 \\
\hline cellular amino acid catabolic process & -2.10 & 0.00 & 0.01 \\
\hline fatty acid catabolic process & -2.01 & 0.00 & 0.01 \\
\hline
\end{tabular}

\section{HPA validation}

we further analyzed the protein level of TLCD1 in clinical liver tissues from HPA database. The results of immunohistochemical indicated that the protein expression level of TLCD1 significantly abnormal between normal tissues and HCC tissues. Immunohistochemistry analysis available from the HPA showed that in tumor tissues, TLCD1 has higher levels of expression compared to non-tumor tissues (Fig. 6C).

\section{Discussion}

TLCD1 is a gene only reported in membrane fluidity. Firstly, the variations in TLCD1 expression level related to prognosis and infiltration of immune cells in HCC were determined. Responding to the hormonal signals, the major organ, the liver controls the glucose and the lipid metabolism ${ }^{29}$. The action of TLCD1 at the level of the plasma membrane by limiting the amounts of LCPUFA-containing 
phospholipids was suggested by the previous study ${ }^{7}$. In the maintenance of the structure and function of the cell membrane and cancer metabolism the polyunsaturated fatty acids played a significant role ${ }^{30,31}$. When lipid metabolism and fatty acid catabolic are disorder, a series of pathological changes will occur in the liver. Our studies suggested that TLCD1 could be used as a promising cancer biomarker in HCC as it was found to be having a potential influence on tumor immunology.

During our current research, the expression of TLCD1 as a prognostic biomarker in HCC was first explored. All data of HCC patients downloaded from TCGA were performed to estimate the prognostic value. From the perspective of clinical pathology, tumor-infiltrating immune cells and biological functions, it was observed that the up-regulated TLCD1 was an independent prognostic factor for the overall poor survival rate. The liver cancer patients with high TLCD1 expression were found to be more susceptible to a more advanced tumor, grade, and stage status against the low expression of TLCD1. The potential influences of high TLCD1 expression levels on the mechanisms of tumor immunology and tumorigenesis in HCC progression were proposed by our results. For human HCC prognosis, the TLCD1 could serve as a predictor.

The correlation between the diverse immune infiltration levels and TLCD1 expression in liver cancer was another important aspect of our study. The study also demonstrated that the infiltration levels of immune cells in HCC could be detected with TIMER. The outcomes revealed that TLCD1 had strongest relationships with $B$ cells, macrophage and dendritic cells. Besides, CIBERSORT confirmed the presence of a moderate to strong positive relationships between the infiltration levels of immune cells and the TLCD1 expression, especially Tregs and dendritic cells. The results in our study could indicate correlation between possible mechanism where TLCD1 regulates Tregs functions in HCC. Regulatory T cells contributes to failure of T cell-mediated immunity ${ }^{32}$. Through cytokine secretion and via cell-to-cell contact, Tregs suppress activation and differentiation of many cell type and sustain tolerance to selfantigens and regulate the immune system ${ }^{33}$. There are opinions that Tregs have a central role in emergence of HCC persistence. We investigated TLCD1 has a strong effect on gene (TNFRSF18 ${ }^{34}$, IL1R1, IL1R2 ${ }^{35}, \mathrm{JAK}^{36}{ }^{36}, \mathrm{CTLA}^{37}{ }^{37}, \mathrm{TNFRSF}^{38}$ and CD $274^{39}$ ) expression related to lipid metabolism.

GO term and KEGG pathway analysis in this study revealed that the up-regulated TLCD1 to be primarily linked with fatty acid catabolic process and PPAR signaling pathway. Lipid metabolism, including fatty acid catabolic, is a primary function of the liver ${ }^{40}$. Our study here implicated that overexpression of TLCD1 in HCC patients could induce lipid accumulation and disorder of lipid metabolism. Further studies are needed to confirm if and how TLCD1 supports HCC metastasis in vivo. Evidently, our results could establish the development in the field of TLCD1 biological function in promoting the motility of the HCC cancer cells.

\section{Conclusion}

Our study was first to identify TLCD1 as a new biomarker of the hepatocellular carcinoma thereby helping to determine how the TME cells and the fatty acid catabolic process could promote the development of 
liver cancer. In HCC studies, it could be a brand-new biomarker. The biomarker therapies could become a promising future option in the treatment of liver diseases with a better understanding of the functional diversity and heterogeneity of TLCD1. An effective design of the therapeutic strategies and diagnosis for treating human HCC could be contributed by TLCD1.

\section{Abbreviations}

HCC: hepatocellular carcinoma, TCGA: The Cancer Genome Atlas, AFP: alpha-fetoprotein, TLCD1: TLC Domain Containing 1, LCPUFA: Long-chain Polyunsaturated Fatty Acids, GEPIA: Expression Profiling Interactive Analysis, GSEA: Gene Set Enrichment Analysis, GO: Gene Ontology, KEGG: Kyoto Encyclopedia of Genes and Genomes

\section{Declarations}

\section{Acknowledgements}

None

\section{Authors' contributions}

HY-S, ZH-W, YN-D, DD-Z, AL-H came up with the design and conception. The data analysis and visualization were conducted by HY-S, SQ-R, L-D, JL-Z and W-W. The original writing of the draft and its editing were by HY-S, SQ-R, PP-S, ZH-W and YN-D. All authors wrote and reviewed the manuscript, and finally approved the submitted manuscript

\section{Funding}

This study was funded by the National Natural Science Foundation of China, China (No. 81871677) and Postgraduate Research \& Practice Innovation Program of Jiangsu Province (No. KYCX20_2839).

\section{Availability of data and materials}

The data was downloaded from TCGA database.

\section{Ethics approval and consent to participate}

Not applicable.

\section{Consent for publication}




\section{Competing interests}

The authors declare that they have no competing interests.

\section{Conflicts of interests:}

None

\section{References}

1. Gong D-Y, Chen E-Q, Huang F-J, Leng X-H, Cheng X, Tang H. Role and Functional Domain of Hepatitis $B$ Virus X Protein in Regulating HBV Transcription and Replication in Vitro and in Vivo. Viruses. 2013.

2. Fan $X$, Wang P, Sun Y, et al. Induction of apoptosis by an oleanolic acid derivative in SMMC-7721 human hepatocellular carcinoma cells is associated with mitochondrial dysfunction. Oncol Lett. 2018;15(3):2821-2828.

3. Thein HH, Qiao Y, Zaheen A, et al. Cost-effectiveness analysis of treatment with non-curative or palliative intent for hepatocellular carcinoma in the real-world setting. PLoS One. 2017;12(10):e0185198.

4. Mehta N, Dodge JL, Grab JD, Yao FY. National Experience on Down-Staging of Hepatocellular Carcinoma Before Liver Transplant: Influence of Tumor Burden, Alpha-Fetoprotein, and Wait Time. Hepatology. 2020;71(3):943-954.

5. Zhang D, Ma X, Sun W, Cui P, Lu Z. Down-regulated FSTL5 promotes cell proliferation and survival by affecting Wnt/beta-catenin signaling in hepatocellular carcinoma. Int J Clin Exp Pathol. 2015;8(3):3386-3394.

6. Papanayotou C, De Almeida I, Liao P, et al. Calfacilitin is a calcium channel modulator essential for initiation of neural plate development. Nat Commun. 2013;4:1837.

7. Ruiz M, Bodhicharla R, Svensk E, et al. Membrane fluidity is regulated by the $C$. elegans transmembrane protein FLD-1 and its human homologs TLCD1/2. Elife. 2018;7.

8. Bannenberg G, Mallon C, Edwards H, et al. Omega-3 Long-Chain Polyunsaturated Fatty Acid Content and Oxidation State of Fish Oil Supplements in New Zealand. Sci Rep. 2017;7(1):1488.

9. Castro LF, Tocher DR, Monroig O. Long-chain polyunsaturated fatty acid biosynthesis in chordates: Insights into the evolution of Fads and Elovl gene repertoire. Prog Lipid Res. 2016;62:25-40.

10. Zhu KC, Song L, Guo HY, et al. Elovl4a participates in LC-PUFA biosynthesis and is regulated by PPARalphabeta in golden pompano Trachinotus ovatus (Linnaeus 1758). Sci Rep. 2019;9(1):4684.

11. Park JB, Lee CS, Jang JH, et al. Phospholipase signalling networks in cancer. Nature reviews Cancer. 2012;12(11):782-792. 
12. Joyce JA, Pollard JW. Microenvironmental regulation of metastasis. Nature reviews Cancer. 2009;9(4):239-252.

13. Beloribi-Djefaflia S, Vasseur S, Guillaumond F. Lipid metabolic reprogramming in cancer cells. Oncogenesis. 2016;5:e189.

14. Baenke F, Peck B, Miess H, Schulze A. Hooked on fat: the role of lipid synthesis in cancer metabolism and tumour development. Disease models \& mechanisms. 2013;6(6):1353-1363.

15. Smith RE, Lespi P, Di Luca M, et al. A reliable biomarker derived from plasmalogens to evaluate malignancy and metastatic capacity of human cancers. Lipids. 2008;43(1):79-89.

16. Doria ML, Cotrim Z, Macedo B, et al. Lipidomic approach to identify patterns in phospholipid profiles and define class differences in mammary epithelial and breast cancer cells. Breast Cancer Res Treat. 2012;133(2):635-648.

17. Hilvo M, Denkert C, Lehtinen L, et al. Novel theranostic opportunities offered by characterization of altered membrane lipid metabolism in breast cancer progression. Cancer Res. 2011;71(9):3236-3245.

18. Fhaner CJ, Liu S, Ji H, Simpson RJ, Reid GE. Comprehensive lipidome profiling of isogenic primary and metastatic colon adenocarcinoma cell lines. Anal Chem. 2012;84(21):8917-8926.

19. Bolouri H. Network dynamics in the tumor microenvironment. Seminars in cancer biology. 2015;30:52-59.

20. Jia D, Li S, Li D, Xue H, Yang D, Liu Y. Mining TCGA database for genes of prognostic value in glioblastoma microenvironment. Aging. 2018;10(4):592-605.

21. Wang Z, Jensen MA, Zenklusen JC. A Practical Guide to The Cancer Genome Atlas (TCGA). Methods Mol Biol. 2016;1418:111-141.

22. Tang Z, Li C, Kang B, Gao G, Li C, Zhang Z. GEPIA: a web server for cancer and normal gene expression profiling and interactive analyses. Nucleic acids research. 2017;45(W1):W98-w102.

23. Yoshihara K, Shahmoradgoli M, Martínez E, et al. Inferring tumour purity and stromal and immune cell admixture from expression data. Nature communications. 2013;4:2612.

24. Li B, Severson E, Pignon JC, et al. Comprehensive analyses of tumor immunity: implications for cancer immunotherapy. Genome Biol. 2016;17(1):174.

25. Aran D, Sirota M, Butte AJ. Systematic pan-cancer analysis of tumour purity. Nat Commun. 2015;6:8971.

26. Subramanian A, Tamayo P, Mootha VK, et al. Gene set enrichment analysis: a knowledge-based approach for interpreting genome-wide expression profiles. Proceedings of the National Academy of Sciences of the United States of America. 2005;102(43):15545-15550.

27. Lanczky A, Nagy A, Bottai G, et al. miRpower: a web-tool to validate survival-associated miRNAs utilizing expression data from 2178 breast cancer patients. Breast cancer research and treatment. 2016;160(3):439-446.

28. Ohtani H. Focus on TILs: prognostic significance of tumor infiltrating lymphocytes in human colorectal cancer. Cancer immunity. 2007;7:4. 
29. Qiu X, Li J, Lv S, et al. HDAC5 integrates ER stress and fasting signals to regulate hepatic fatty acid oxidation. J Lipid Res. 2018;59(2):330-338.

30. Mikami K, Murata N. Membrane fluidity and the perception of environmental signals in cyanobacteria and plants. Prog Lipid Res. 2003;42(6):527-543.

31. Zhang F, Du G. Dysregulated lipid metabolism in cancer. World J Biol Chem. 2012;3(8):167-174.

32. Accapezzato D, Francavilla V, Paroli M, et al. Hepatic expansion of a virus-specific regulatory CD8(+) T cell population in chronic hepatitis C virus infection. J Clin Invest. 2004;113(7):963-972.

33. Thompson C, Powrie F. Regulatory T cells. Curr Opin Pharmacol. 2004;4(4):408-414.

34. Passerini L, Barzaghi F, Curto R, et al. Treatment with rapamycin can restore regulatory T-cell function in IPEX patients. The Journal of allergy and clinical immunology. 2020;145(4):1262-1271.e1213.

35. Ritvo PG, Churlaud G, Quiniou V, et al. T(fr) cells lack IL-2Ra but express decoy IL-1R2 and IL-1Ra and suppress the IL-1-dependent activation of T(fh) cells. Science immunology. 2017;2(15).

36. Hossain DM, Panda AK, Chakrabarty S, et al. MEK inhibition prevents tumour-shed transforming growth factor- $\beta$-induced T-regulatory cell augmentation in tumour milieu. Immunology. 2015;144(4):561-573.

37. Ohkura N, Sakaguchi S. Transcriptional and epigenetic basis of Treg cell development and function: its genetic anomalies or variations in autoimmune diseases. Cell research. 2020;30(6):465-474.

38. Jacquemin C, Augusto JF, Scherlinger M, et al. OX40L/OX40 axis impairs follicular and natural Treg function in human SLE. JCl insight. 2018;3(24).

39. Di Pilato M, Kim EY, Cadilha BL, et al. Targeting the CBM complex causes $T$ (reg) cells to prime tumours for immune checkpoint therapy. Nature. 2019;570(7759):112-116.

40. Yan G, Li X, Peng Y, et al. The Fatty Acid beta-Oxidation Pathway is Activated by Leucine Deprivation in HepG2 Cells: A Comparative Proteomics Study. Sci Rep. 2017;7(1):1914.

\section{Figures}


A

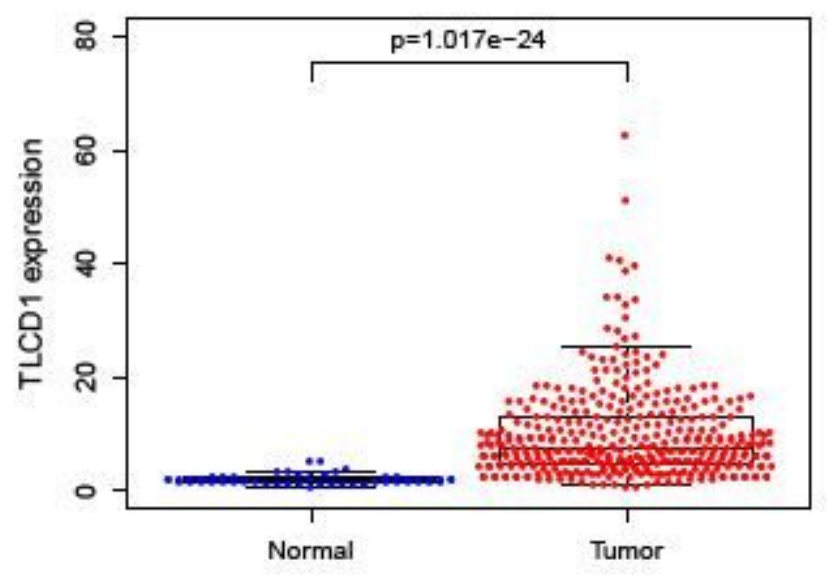

C

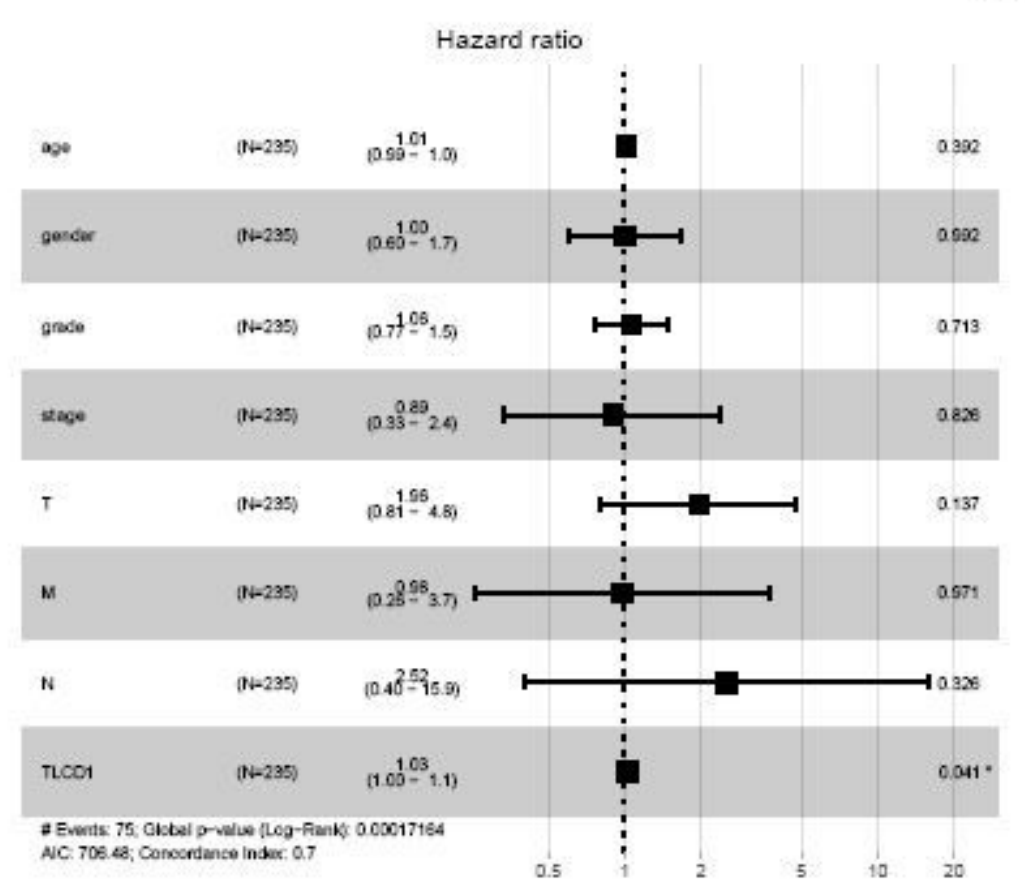

B

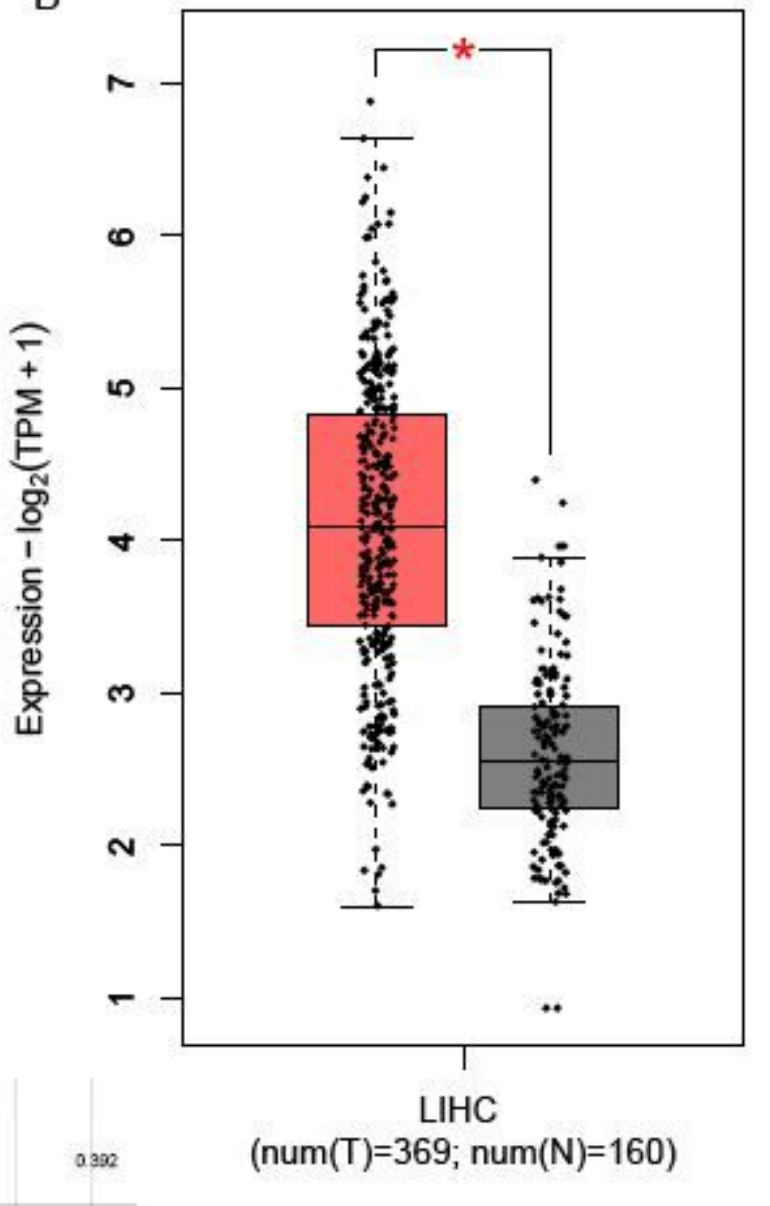

\section{Figure 1}

(A)The expression of TLCD1 between normal and tumor tissues in TCGA (B) TLCD1 mRNA expression levels in normal and HCC tissues, as obtained from GEPIA (C)Multivariate Cox analysis of TLCD1 expression and other clinicopathological variables 
A
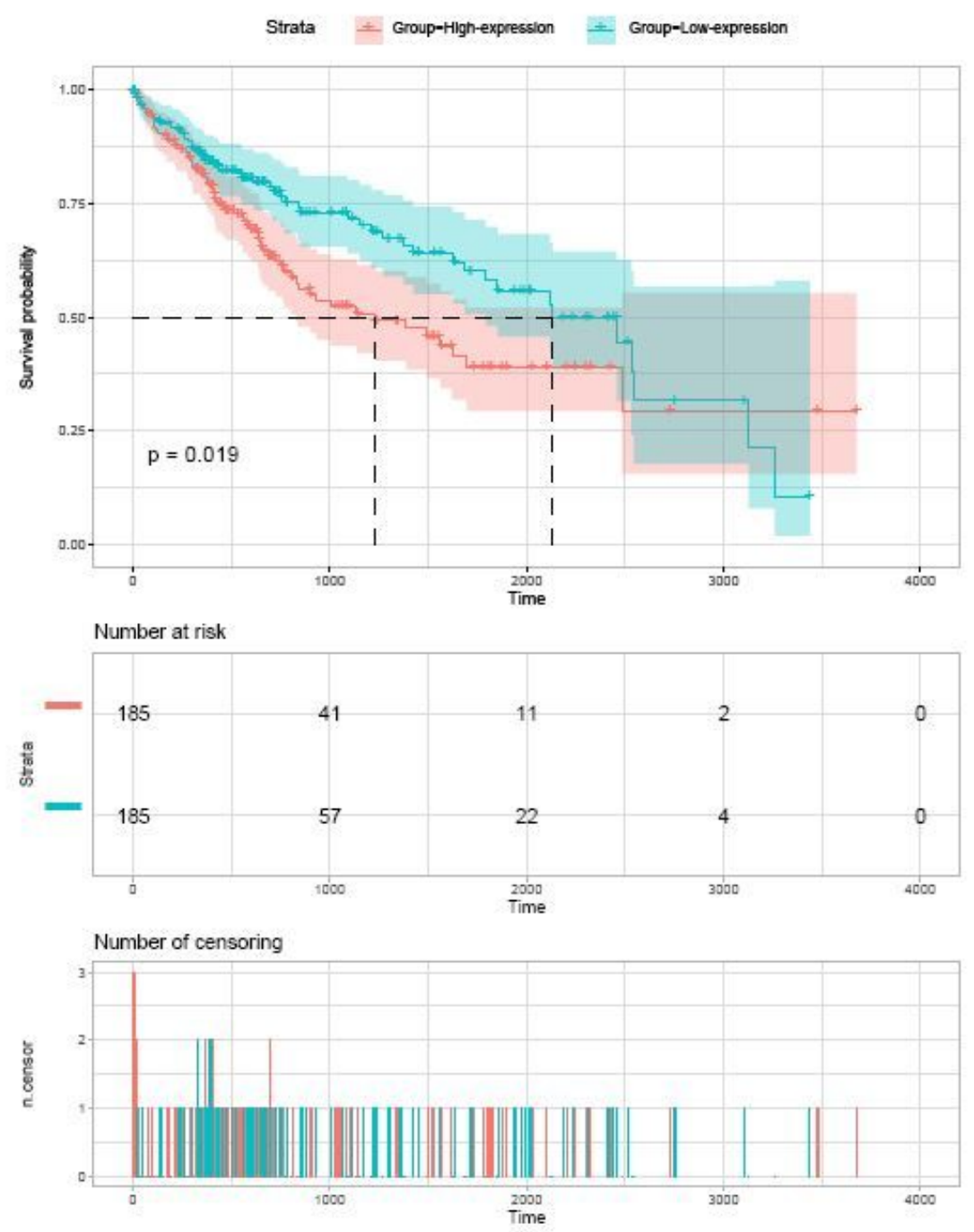

B

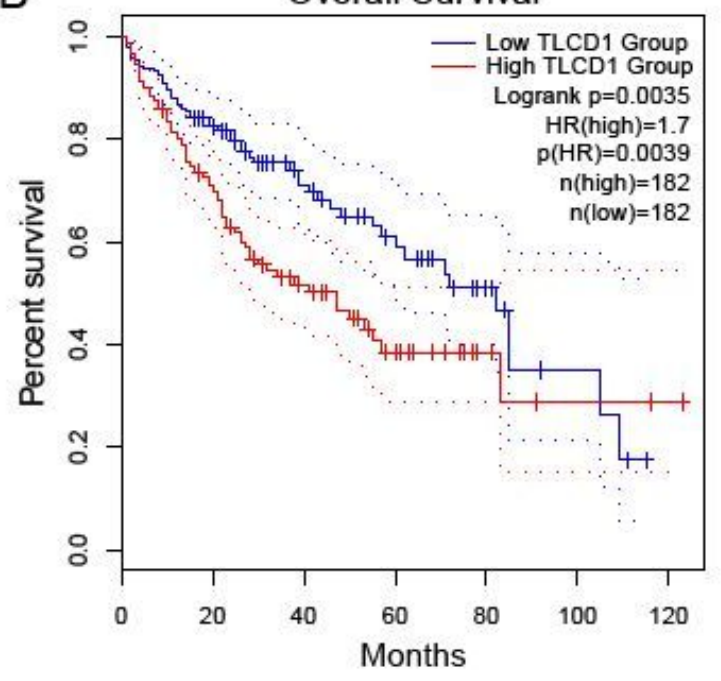

C
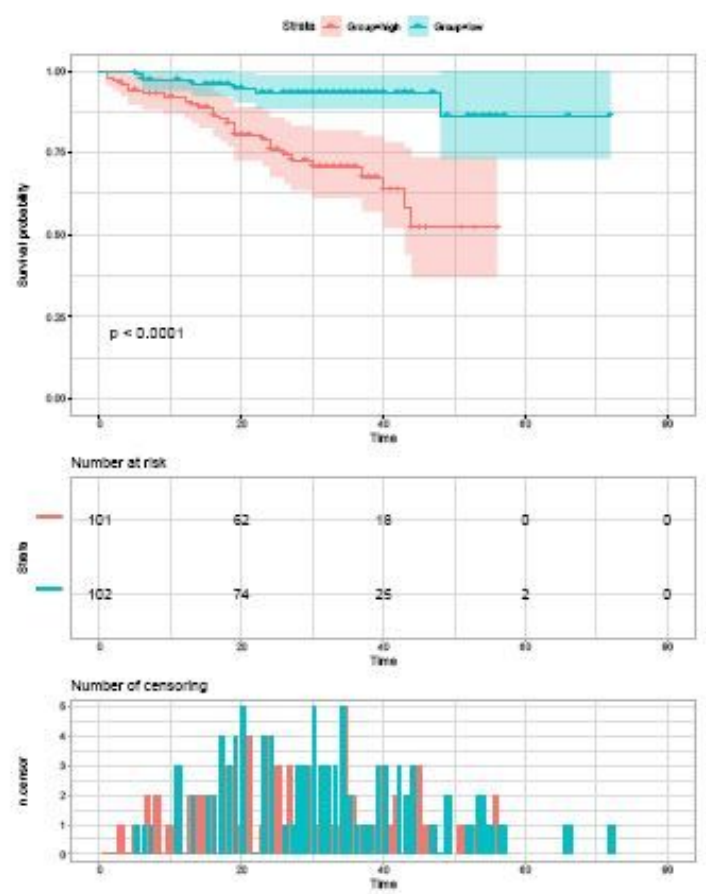

\section{Figure 2}

Kaplan-Meier analyses of TLCD1 expression for patient survival. (A)TCGA database (B)GEPIA database (C)ICGC database 

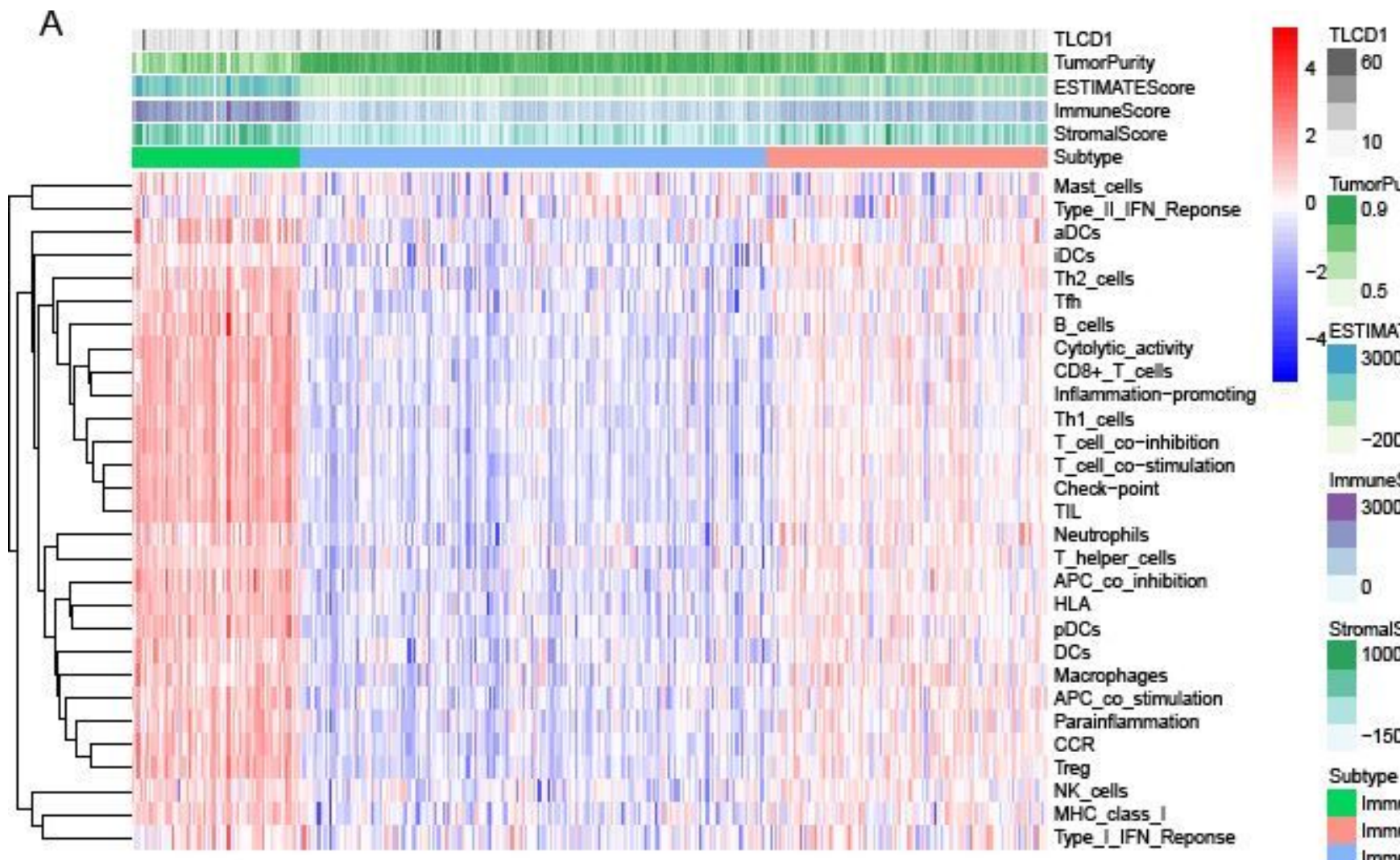

TumorPunity

0.9

$\begin{array}{ll}-2 & 0.5\end{array}$

-4 ESTIMATEScore

3000

$-2000$

ImmuneScore
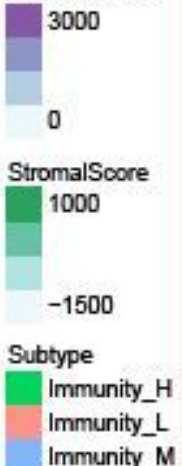

B

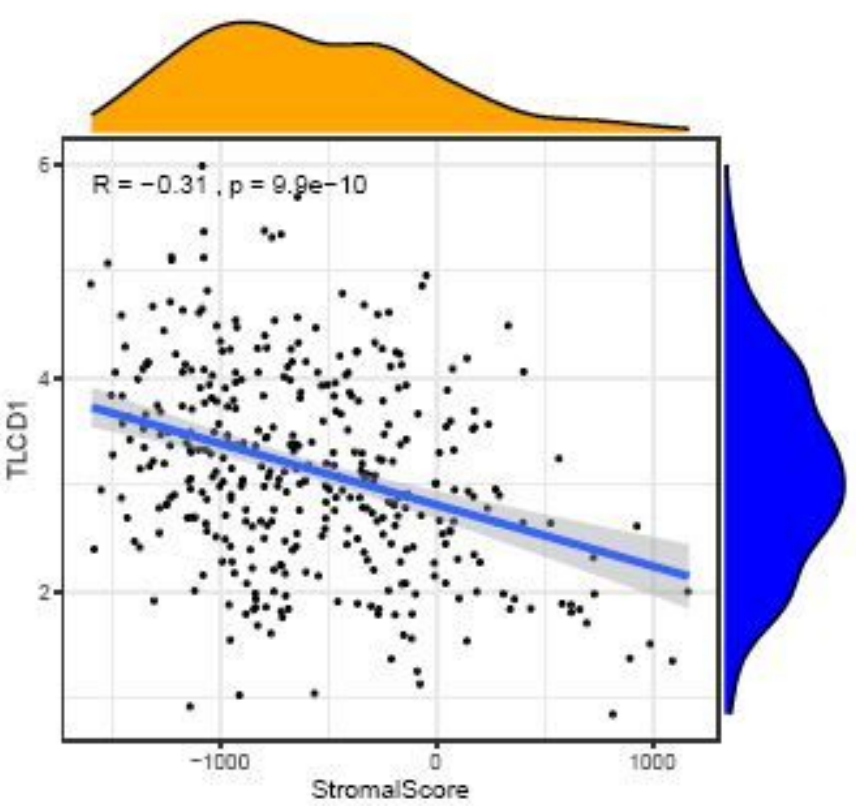

C

\section{Cancer: LIHC}

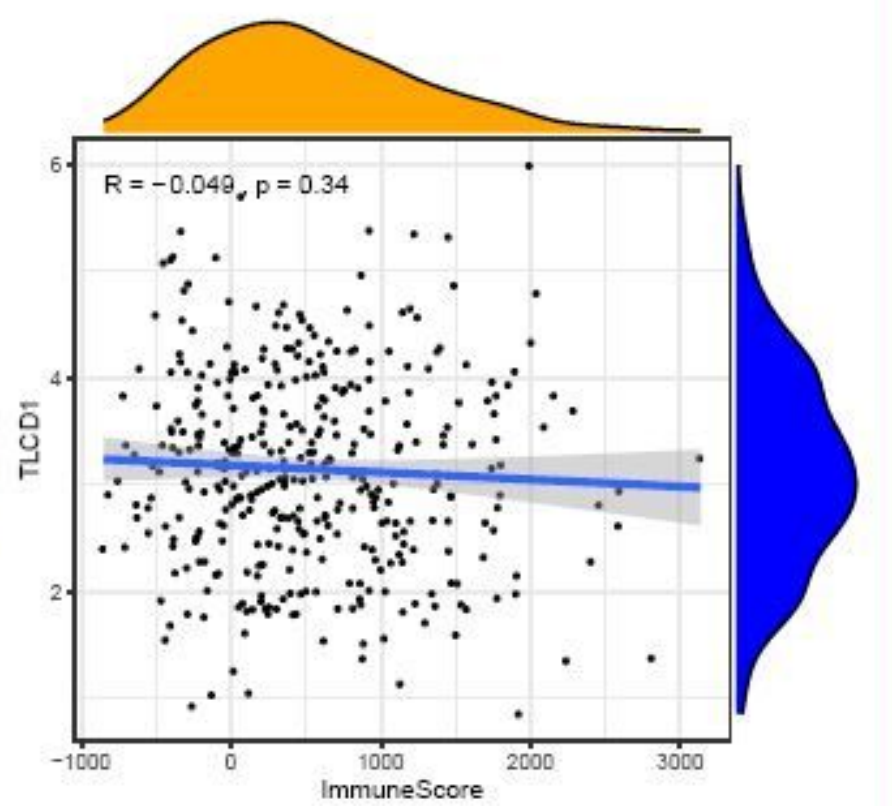

Figure 3

(A) Hierarchical clustering heatmap of each cell infiltration in the HCC (B) The relationships between TLCD1 expression and stromal score (C) The relationships between TLCD1 expression and immune cells 
A
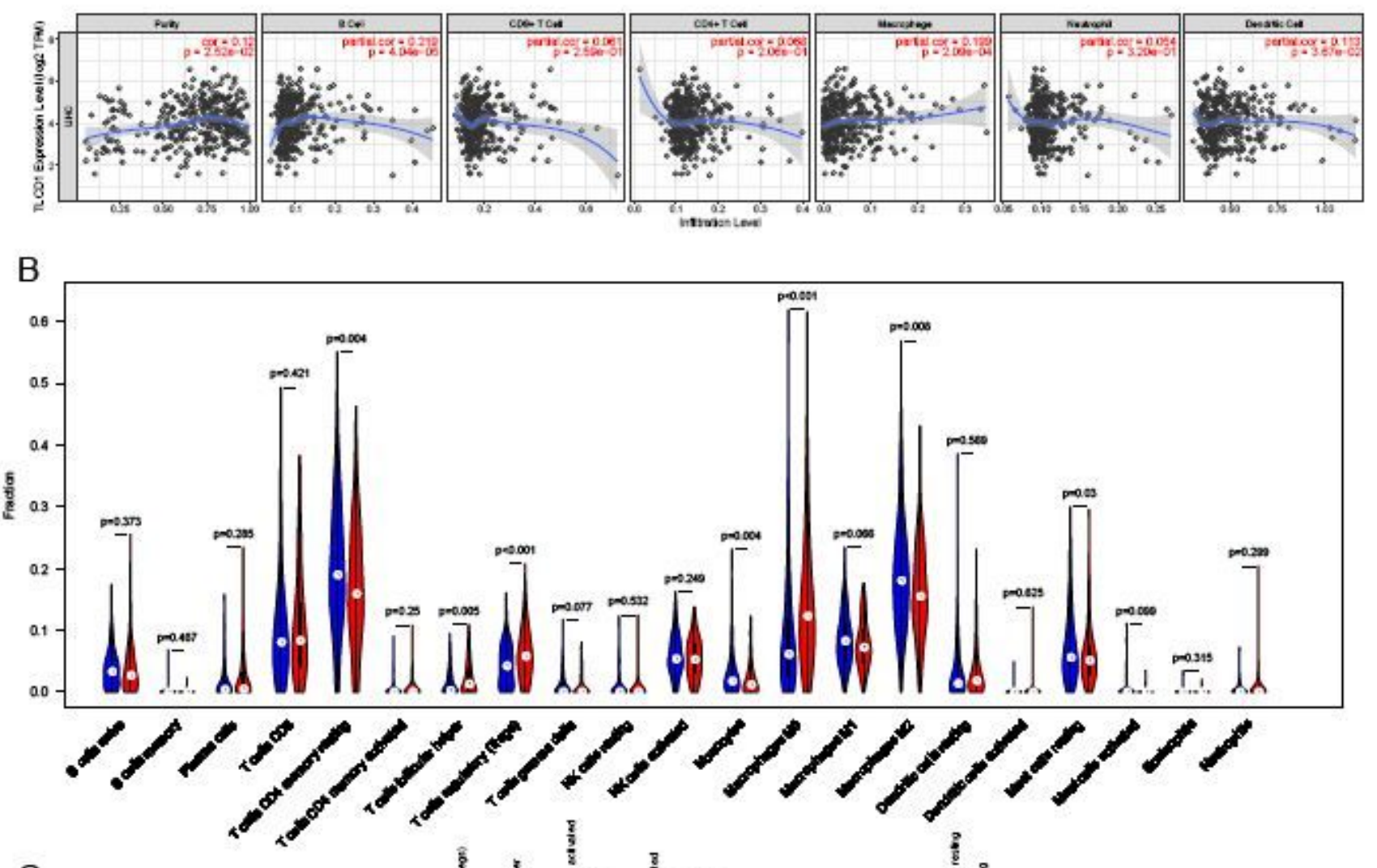

C

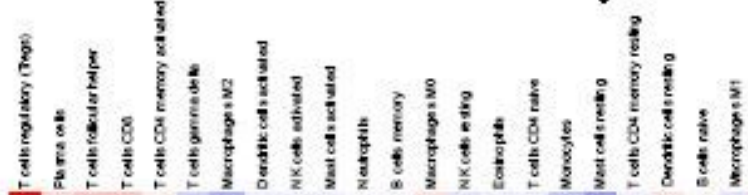

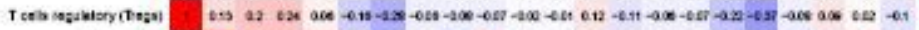

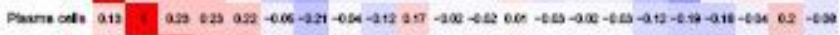

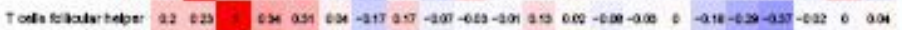

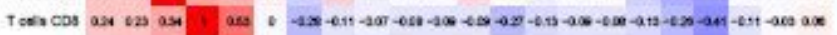

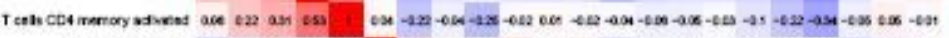

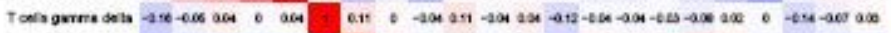

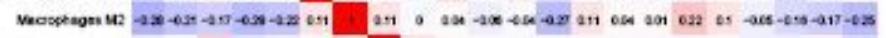

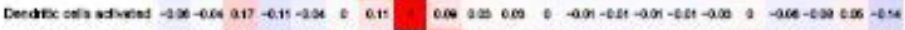

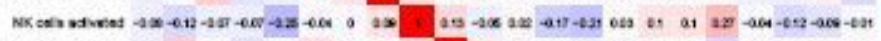

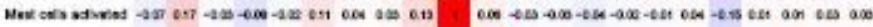

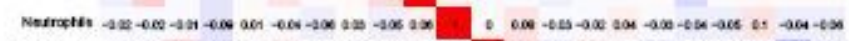

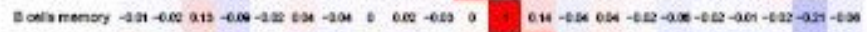

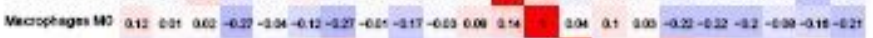

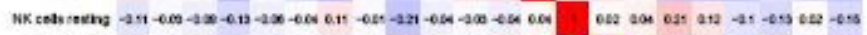

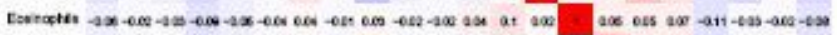

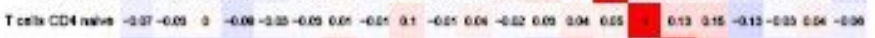

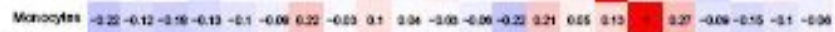

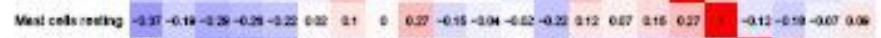

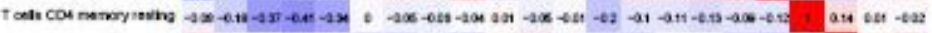

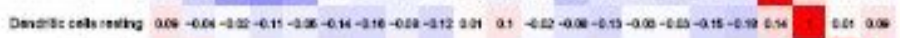

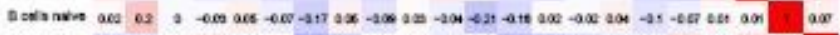

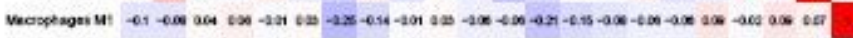

\section{Figure 4}

(A) Correlations between TLCD1 expression and immune infiltration levels (B) The varied proportions of 22 subtypes of immune cells in high and low TLCD1 expression groups in tumor samples (C) Heatmap of 22 immune infiltration cells in tumor samples. 
A

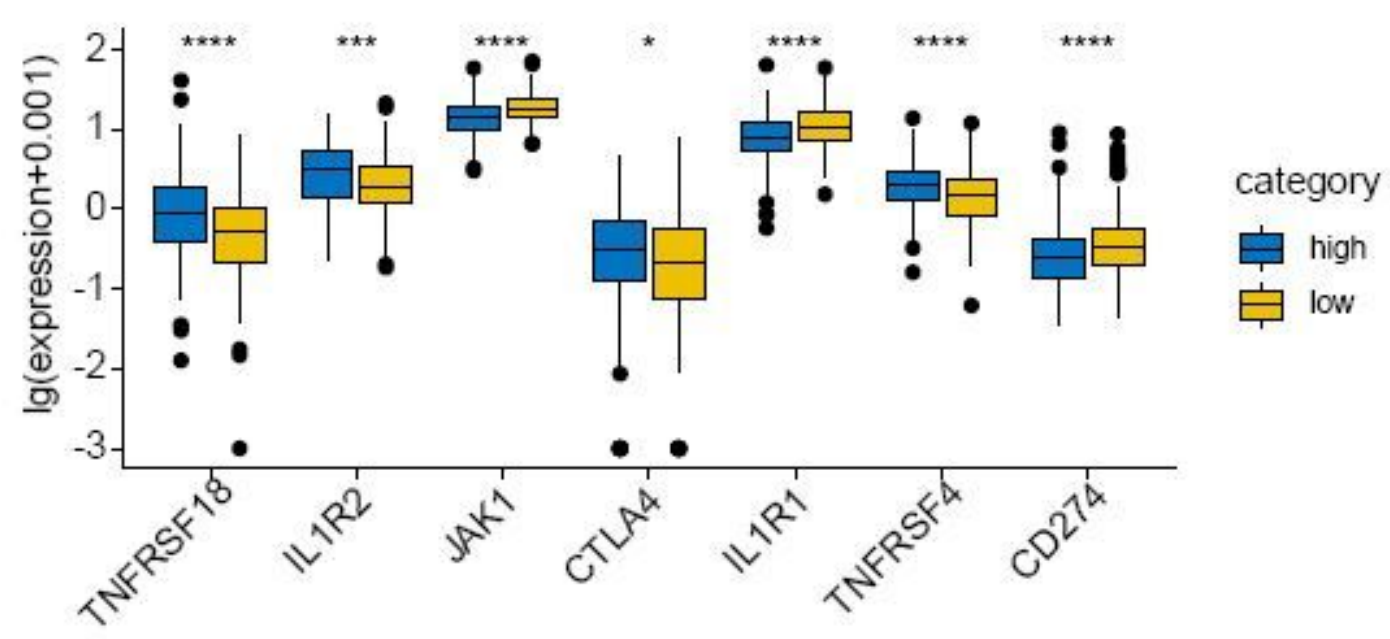

B

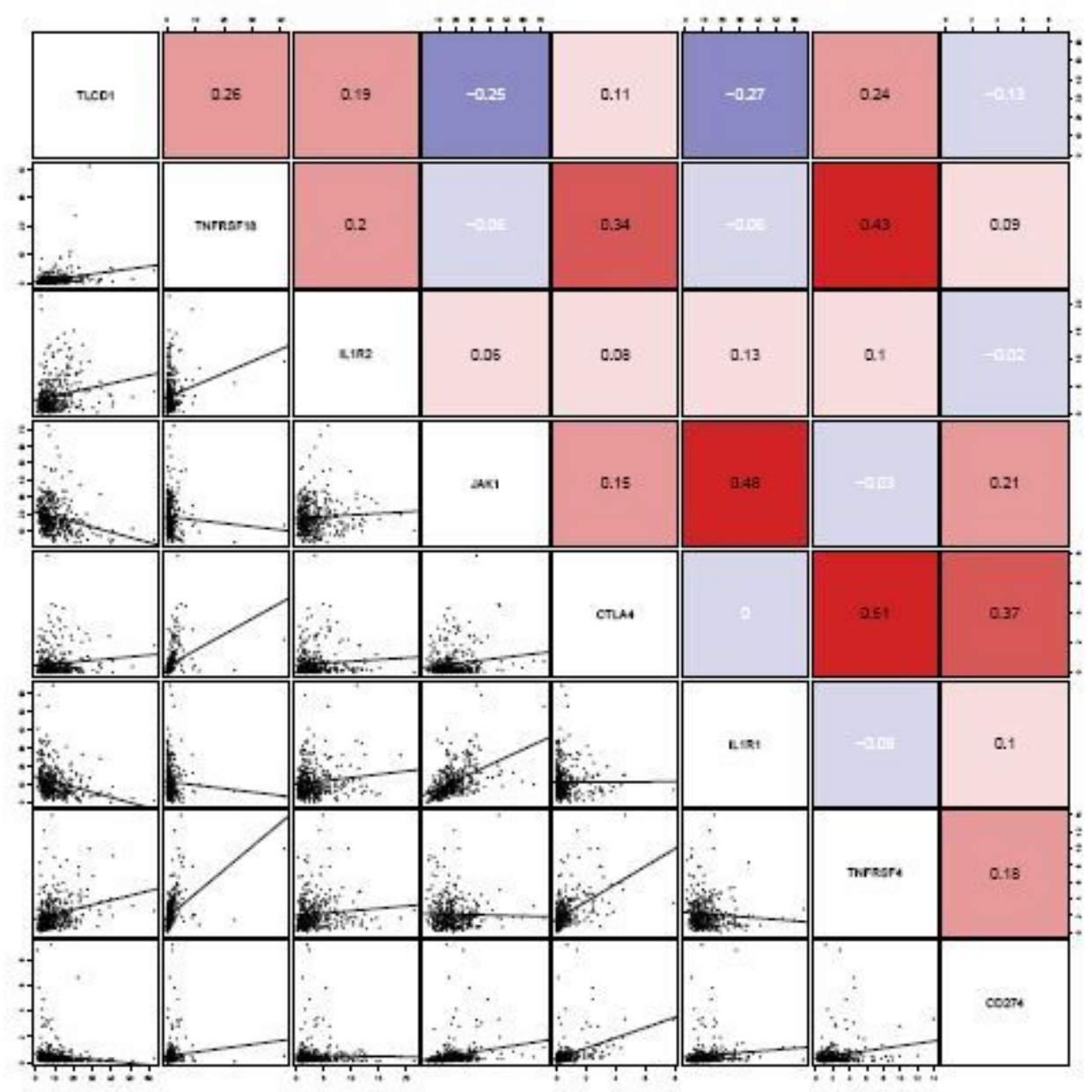

Figure 5

(A) Box plot of metabolic genes and TLCD1 expression (B) Correlation between TLCD1 expression and Treg-related biomarkers expression 
A
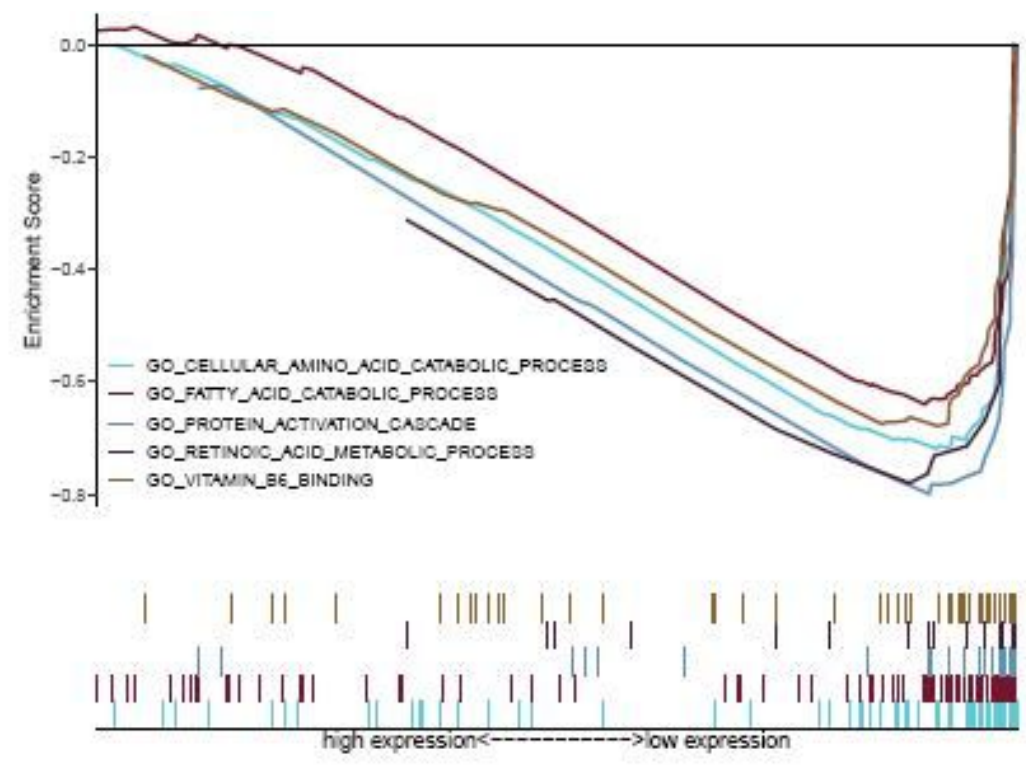

B
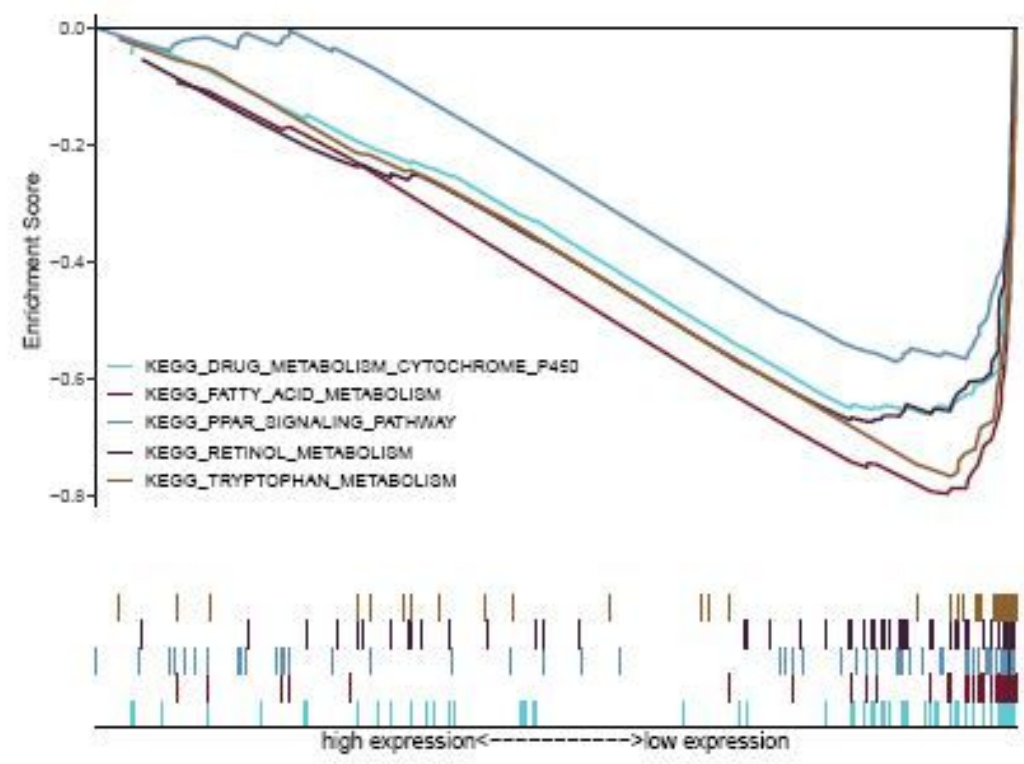

C

$$
\text { normal }
$$

$\mathrm{HCC}$

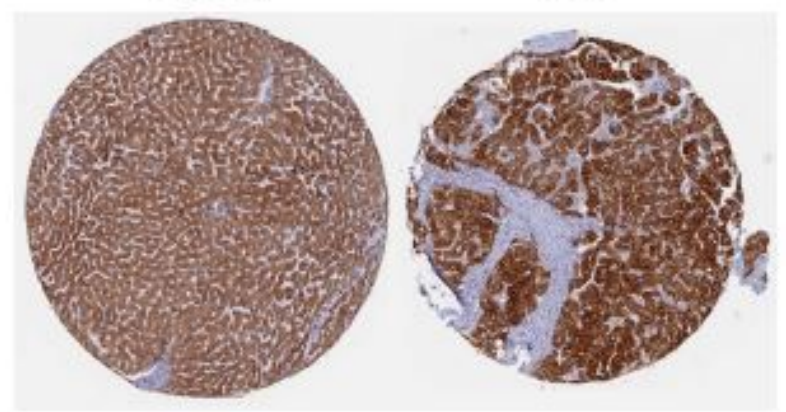

\section{Figure 6}

(A) GO term analysis revealed five correct groups (B) KEGG pathway showed five correlated groups. (C) Representative immunohistochemistry staining results reveal the protein level expression of TLCD1 in HCC and normal tissues in HPA database. 